\title{
No apparent influence of psychometrically-defined schizotypy on orientation-dependent contextual modulation of visual contrast detection
}

\author{
Damien J Mannion ${ }^{\text {Corresp., }}{ }^{1}$, Chris Donkin ${ }^{1}$, Thomas J Whitford ${ }^{1}$ \\ ${ }^{1}$ School of Psychology, UNSW Australia \\ Corresponding Author: Damien J Mannion \\ Email address: d.mannion@unsw.edu.au
}

We investigated the relationship between psychometrically-defined schizotypy and the ability to detect a visual target pattern. Target detection is typically impaired by a surrounding pattern (context) with an orientation that is parallel to the target, relative to a surrounding pattern with an orientation that is orthogonal to the target (orientationdependent contextual modulation). Based on reports that this effect is reduced in those with schizophrenia, we hypothesised that there would be a negative relationship between the relative score on psychometrically-defined schizotypy and the relative effect of orientation-dependent contextual modulation. We measured visual contrast detection thresholds and scores on the Oxford-Liverpool Inventory of Feelings and Experiences ( $\mathrm{O}$ LIFE) from a non-clinical sample $(N=100)$. Contrary to our hypothesis, we find an absence of a monotonic relationship between the relative magnitude of orientation-dependent contextual modulation of visual contrast detection and the relative score on any of the subscales of the O-LIFE. The apparent difference of this result with previous reports on those with schizophrenia suggests that orientation-dependent contextual modulation may be an informative condition in which schizophrenia and psychometrically-defined schizotypy are dissociated. However, further research is also required to clarify the nature of orientation-dependent contextual modulation in those with schizophrenia. 


\title{
No Apparent Influence of Psychometrically-Defined Schizotypy on Orientation-Dependent Contextual Modulation of Visual Contrast Detection
}

\author{
Damien J. Mannion ${ }^{1}$, Chris Donkin ${ }^{1}$, and Thomas J. Whitford ${ }^{1}$ \\ ${ }^{1}$ School of Psychology, UNSW Australia
}

\begin{abstract}
We investigated the relationship between psychometrically-defined schizotypy and the ability to detect a visual target pattern. Target detection is typically impaired by a surrounding pattern (context) with an orientation that is parallel to the target, relative to a surrounding pattern with an orientation that is orthogonal to the target (orientation-dependent contextual modulation). Based on reports that this effect is reduced in those with schizophrenia, we hypothesised that there would be a negative relationship between the relative score on psychometrically-defined schizotypy and the relative effect of orientation-dependent contextual modulation. We measured visual contrast detection thresholds and scores on the OxfordLiverpool Inventory of Feelings and Experiences (O-LIFE) from a non-clinical sample $(N=100)$. Contrary to our hypothesis, we find an absence of a monotonic relationship between the relative magnitude of orientation-dependent contextual modulation of visual contrast detection and the relative score on any of the subscales of the O-LIFE. The apparent difference of this result with previous reports on those with schizophrenia suggests that orientation-dependent contextual modulation may be an informative condition in which schizophrenia and psychometrically-defined schizotypy are dissociated. However, further research is also required to clarify the nature of orientation-dependent contextual modulation in those with schizophrenia.
\end{abstract}

Keywords:

\section{INTRODUCTION}

Because visual processing appears to differ in those with and without a diagnosis of schizophrenia (Butler et al., 2008), increased knowledge of vision in schizophrenia can provide important progress towards our understanding of the disorder (Yoon et al., 2013). The concept of 'schizotypy' proposes that schizophrenia characterises the far end of a continuum of symptomatology that varies across the general population (Lenzenweger, 2010; Mason and Claridge, 2015). There is growing empirical evidence for the validity of the construct of schizotypy; for example, non-clinical individuals scoring highly on psychometric measures of schizotypy have been shown to exhibit cognitive, behavioural, and neurophysiological abnormalities similar to those observed in patients with established schizophrenia (Oestreich et al., 2015; Asai et al., 2008; Lenzenweger and O'Driscoll, 2006; Modinos et al., 2010). This raises the possibility that advances in understanding schizophrenia can be made by examining how perceptual processing changes in non-clinical individuals who exhibit high levels of schizotypy (Ettinger et al., 2015).

However, there have been relatively few investigations into the relationship between visual perception and schizotypy. Rawlings and Claridge (1984) reported that those that are rated highly on schizotypy show differences in visual field asymmetries (left/right) on letter recognition and local/global tasks. Koychev et al. (2010) found the early visual evoked potential to be reduced in those rated highly on schizotypy. Schizotypy has also been reported to be associated with differences in visual backward masking (Cappe et al., 2012) and depth processing (Barbato et al., 2012), although differences were limited to a particular schizotypy dimension and task, respectively. There have also been reports of an absent relationship between schizotypy and performance on perceptual organisation (Silverstein et al., 1992) and context effects on size and contour integration (Uhlhaas et al., 2004). 
Visual contextual modulation, in which the perception of a target stimulus is affected by the presence of a surrounding stimulus, has received considerable investigation in those with schizophrenia (Barch et al., 2012; Dakin et al., 2005; Tibber et al., 2013; Yang et al., 2013, for example). The orientation-dependent surround effect is a specific instance of contextual modulation that has been used to examine visual processing in those with schizophrenia (Schallmo et al., 2015; Serrano-Pedraza et al., 2014; Seymour et al., 2013; Yoon et al., 2009), and is particularly relevant due to its well-established behavioural and neural foundations. In this approach, a target pattern is surrounded by a similar pattern of either the same orientation (parallel) or the orthogonal orientation. For example, a vertical target would be surrounded by either a vertical (parallel) or horizontal (orthogonal) contextual pattern. In observers without schizophrenia, the presence of parallel context affects visual processing of the target to a much greater extent than orthogonal context (Cannon and Fullenkamp, 1991; Petrov et al., 2005; Zenger-Landolt and Heeger, 2003). However, observers with schizophrenia are reported to be relatively less affected by the presence of a parallel compared to an orthogonal surround (Serrano-Pedraza et al., 2014; Seymour et al., 2013; Yoon et al., 2009, 2010; though see Schallmo et al., 2015, and the Discussion).

Here, our primary aim was to examine whether such apparent alterations in visual processing in those with schizophrenia also affect those with relatively high levels of psychometrically-defined schizotypy. We adapted the paradigm of Serrano-Pedraza et al. (2014) to measure contrast detection thresholds for vertical patterns with parallel (vertical) and orthogonal (horizontal) surrounding context. We hypothesised that increased levels of psychometrically-defined schizotypy would be negatively related to the orientation-dependent effect of context on visual contrast detection-that is, increased scores on a psychometric measure of schizotypy would be associated with a relative decrease in the influence of context that is oriented parallel, relative to orthogonal, to a visual target. Consistent with previous reports in schizophrenia (Serrano-Pedraza et al., 2014, for example), we further hypothesised that such a relationship would be attributable to a relative increase in the contrast detection threshold with orthogonal context.

Our additional aim was to advance and test a hypothesis concerning the temporal dynamics of orientation-dependent contextual modulation. Studies on auditory perception have shown that the amplitude of an early event-related potential component in response to a tone is reduced if the tone is elicited by a motor action rather than simply presented in the absence of an eliciting motor action (Schafer and Marcus, 1973). This reduction is weaker in those with schizophrenia (Ford et al., 2001) and in those that score highly on psychometrically-defined schizotypy (Oestreich et al., 2016). Interestingly, imposing a short delay between the motor action and the auditory tone increases the strength of the response reduction in those with schizophrenia (Whitford et al., 2011) and in those with high schizotypy (Oestreich et al., 2016). We considered that a similar phenomenon may operate in orientation-dependent contextual modulation in vision; if temporal processing is altered in those with schizophrenia, simultaneous presentation of target and context may be processed as asynchronous - a situation which strongly reduces orientation-dependent contextual modulation in typical observers (Kilpeläinen et al., 2007; Petrov and McKee, 2009). Hence, we also included a manipulation in which the onset of the surrounding context was $50 \mathrm{~ms}$ before the onset of the target. We predicted that this leading surround would reinstate the orientation-dependent modulation of contrast detection in those that score highly on psychometrically-defined schizotypy.

\section{MATERIALS \& METHODS}

\section{Participants}

Participants $(N=100)$ with normal or corrected-to-normal vision were recruited from a pool of students enrolled in an introductory psychology course at UNSW Australia. Participants received course credit for their involvement and gave informed and written consent in accordance with the experiment protocols approved by the Human Research Ethics Advisory Panel in the School of Psychology, UNSW Australia (2495/153-164). All participants were naïve to the purposes of the experiment.

\section{Stimuli}

The stimuli were similar to those used by Serrano-Pedraza et al. (2014) and consisted of 'context' and four potential 'target' regions. The context was a circular grating that was 20 degrees of visual angle (dva) in diameter with a spatial frequency of 1 cycle/dva, peak contrast of 25\% (ramped smoothly to zero at the edges according to a raised cosine), and of horizontal or vertical orientation. The potential target regions were four circular patches that were each 3 dva in diameter and centred at 5 dva eccentricity from the 
middle of the context, with an opacity that ramped to zero at the edges following a raised cosine profile. On a given presentation, three of the four target regions were set to zero contrast and the remaining region displayed a vertical grating of the same spatial frequency and phase as the context and with a variable non-zero contrast (see Design and procedure). A small fixation marker ( $0.25 \mathrm{dva})$, with a centre of varying luminance and a black edge, was continually present at the centre of the display. Example stimuli are shown in Figure 1.

\section{Apparatus}

The stimuli were presented on one of two identical Display++ LCD monitors (Cambridge Research Systems, Kent, UK) with a spatial resolution of $1920 \times 1080$ pixels, temporal resolution of $120 \mathrm{~Hz}$, and mean luminance of $60 \mathrm{~cd} / \mathrm{m}^{2}$. The monitors each had a 10-bit output resolution and a linear relationship between graphics card signal and luminance. Participants viewed a monitor in one of two darkened rooms at a distance of $52 \mathrm{~cm}$, via a chin rest, for a total angular subtense of $76.6 \times 43.1 \mathrm{dva}$. The experiment was controlled using PsychoPy 1.82.01 (Peirce, 2007) and Python 2.7.10. The code and data from the study is available at https://bitbucket.org/account/user/mannionlab/projects/ schizotypy_visual_contrast.

Schizotypy was assessed via the Oxford-Liverpool Inventory of Feelings and Experiences (O-LIFE; Mason et al., 1995). This is a paper-based questionnaire with 104 items, each requiring a yes/no response, that assess subscales of "unusual experiences", "cognitive disorganisation", "introvertive anhedonia", and "impulsive non-conformity" (see Mason and Claridge, 2006, for the full inventory of items). The "unusual experiences" subscale (30 items) contains items describing perceptual aberrations, magical thinking, and hallucinations, and is conceived as relating to the positive symptoms of psychosis. The "cognitive disorganisation" subscale (24 items) contains items relating to poor attention, concentration, and decision making, and is conceived as relating to the disorganisation and formal thought disorder associated with psychosis. The "introvertive anhedonia" (27 items) subscale contains items relating to anhedonia and avoidance of intimacy, and is conceived as relating to the negative symptoms of schizophrenia. The "impulsive nonconformity" (23 items) subscale contains items relating to impulsive, anti-social, and eccentric forms of behaviour. The O-LIFE has good internal consistency and test-retest reliability (Burch et al., 1998; Mason et al., 1995).

\section{Design and procedure}

The experimental component of the study used a two-way repeated measures design, with relative orientation (parallel, orthogonal) and temporal relationship (simultaneous, leading surround) as factors. The dependent variable was the contrast detection threshold, defined as the contrast required for the spatial location of the target to be identified with $69.25 \%$ accuracy.

The experimental procedure for a given participant was conducted in a single session lasting approximately one hour. The session consisted of a series of runs, where each run measured a single condition. Each condition was measured on three different runs, giving a total of 12 runs in the session. The ordering of conditions across runs was randomised for each participant. There was a self-paced break of at least 30 seconds between each run.

Each run consisted of a series of trials. Each trial began with a 500ms preparatory period in which the centre of the fixation marker was drawn in black and the remainder of the display was at mean luminance. The stimulus period was then active for $150 \mathrm{~ms}$ ( 18 frames), during which the context and target were each presented for $100 \mathrm{~ms}$ (12 frames). If the trial corresponded to the 'simultaneous' level of the temporal relationship factor, both the context and the target were displayed during the latter $100 \mathrm{~ms}$ of the stimulus period $(50-150 \mathrm{~ms})$. The presentation timecourse was thus that neither the context or the target was shown in the first 50ms and then both the context and the target were simultaneously shown for $100 \mathrm{~ms}$. If the trial corresponded to the 'leading surround' level of the temporal relationship factor, the context was displayed from the beginning of the stimulus period $(0-100 \mathrm{~ms})$ and the target was displayed during the latter $100 \mathrm{~ms}$ of the stimulus period $(50-150 \mathrm{~ms})$. The presentation timecourse was thus that the context only was shown in the first 50ms, then both the context and the target were simultaneously shown for the next $100 \mathrm{~ms}$, and then the target only was shown for the next 50ms. The spatial phases of the context and target gratings were set to a common randomised value for each trial. On each trial, the target was randomly presented at one of the four potential spatial locations. During presentation of the target, the centre of the fixation marker was drawn in white. Following the stimulus period, the response period was indicated by drawing the centre of the fixation marker in dark grey and by drawing thin circular outlines corresponding to 

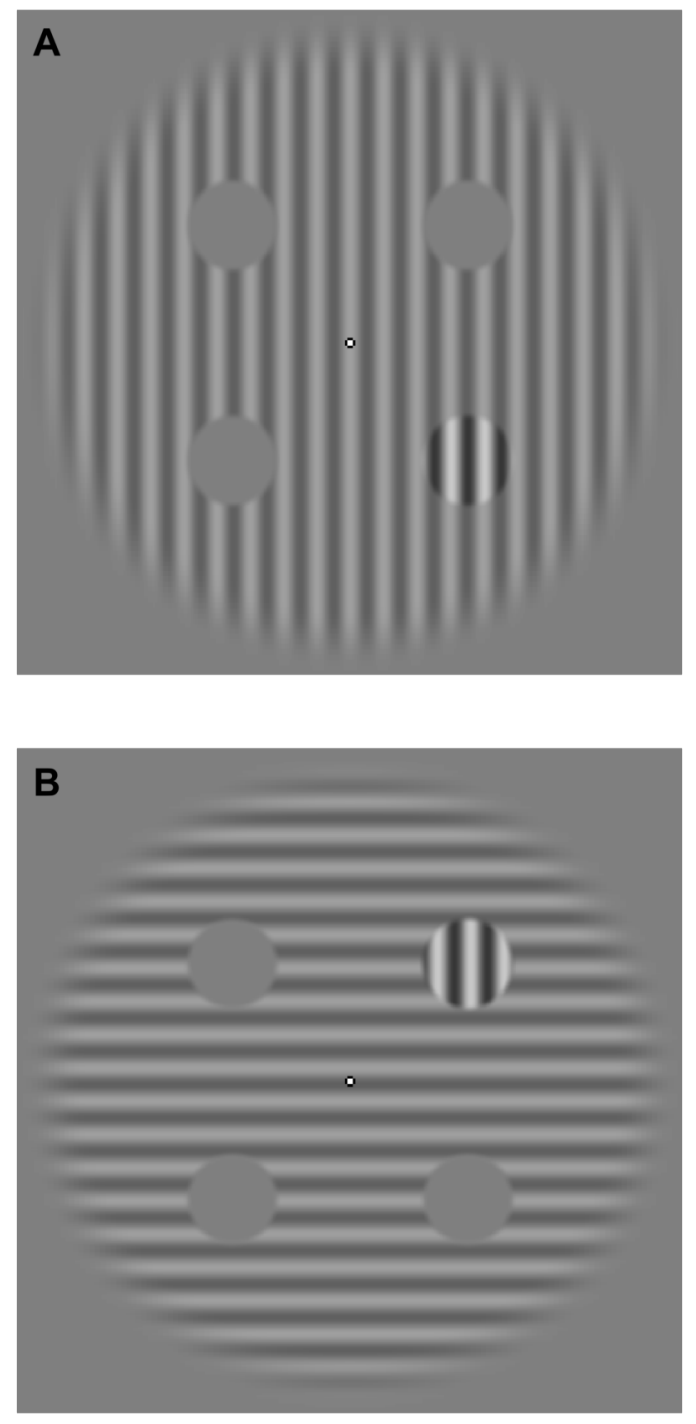

Figure 1. Example stimuli. Panel A depicts the stimulus configuration for the parallel relative orientation condition, with the target in the bottom right quadrant. Panel $\mathbf{B}$ depicts the stimulus configuration for the orthogonal relative orientation condition, with the target in the top right quadrant. 
the positions of the four potential target locations. The participant then indicated their selection using the ' 8 ' (top right), ' 7 ' (top left), '4' (bottom left), or ' 5 ' (bottom right) buttons on a numerical keypad. Feedback was then provided for $200 \mathrm{~ms}$ in the form of a 'tick' (correct) or 'cross' (incorrect) appearing in the centre of the spatial location that contained the target. If necessary, there was then a period of fixation-only presentation to enforce a minimum inter-trial interval of $2 \mathrm{~s}$ before commencing the next trial.

The contrast of the target on each trial was determined using a Psi adaptive staircase procedure (Kontsevich and Tyler, 1999). Here, contrast refers to the Michelson contrast of the grating: $\left(L_{\max }-L_{\min }\right) /\left(L_{\max }+L_{\min }\right)$, where $L_{\max }$ and $L_{\min }$ are the maximum and minimum luminances in the grating, respectively. Each staircase used a Weibull function to capture participant performance, parametrised following Lu and Dosher (2014) as:

$$
P(c)=\zeta+(1-\zeta-\lambda)\left(1-e^{-(c / \tau)^{\eta}}\right)
$$

This psychometric function describes the probability of a correct response for a given target contrast $(c)$, where $\zeta$ is the chance performance level (fixed at 0.25 due to the four alternatives), $\lambda$ is the lapse rate (fixed at 0.05 ), $\tau$ is the threshold (the contrast corresponding to $69.25 \%$ correct performance), and $\eta$ is the slope. For the Psi procedure, the candidate contrast levels were between $0.1 \%$ and $100 \%$ in 350 logarithmicallyspaced values. This distribution was also used for the threshold $(\tau)$, while the slope $(\eta)$ was given by 50 logarithmically-spaced levels between 0.5 and 20 . There were two interleaved staircases on each run, each 40 trials in length, in randomised order across the run. Interleaved staircases were used to improve the robustness of the sampled target contrasts by reducing the opportunity for the algorithm to settle into a narrow range of contrast levels.

Before commencing the experiment, participants were introduced to the task via a set of computerbased instructions. They then completed a practice run in which the context was not present. At the conclusion of the practice run, the experimenter visually evaluated the resulting psychometric functions to determine whether participants understood the task requirements. The practice run was repeated if necessary.

Following completion of the visual task, participants completed the O-LIFE questionnaire and provided basic demographic information (complete set: gender, age, and handedness). Items on the O-LIFE questionnaire were occasionally either unanswered (1 response), answered with both response options ( 2 responses across 1 participant), or ambiguously answered ( 3 responses across 3 participants) such items were replaced with the modal value from that subscale for that participant, after any negative scoring.

\section{Analysis}

The experimental procedure produced 960 data points per participant, where each data point specified the contrast of the target and the correctness of the response. With four conditions, this corresponded to 240 data points per condition ( 3 runs per condition $\times 2$ staircases per run $\times 40$ trials per staircase) for each participant. We summarised the data from each participant and condition by maximum-likelihood fitting of a Weibull psychometric function (see Design and procedure) to obtain threshold $(\tau)$ and slope $(\eta)$ values. A depiction of the raw data and the best-fitting psychometric functions are shown for a representative participant in Figure 2, and for each participant and condition in Figure S1.

We then evaluated the validity and reliability of the best-fitting psychometric functions for each participant. We excluded from further analysis those participants where the estimated slope was shallower than two standard deviations away from the mean slope across all participants, for any condition. This resulted in the exclusion of 7 participants, and subsequent analyses are conducted and reported based on the remaining 93 participants. The raw data and best-fitting psychometric functions for excluded participants are shown in Figure S1.

\section{RESULTS}

Our objective was to investigate the relationship between psychometrically-defined schizotypy and the orientation-dependent modulation of visual contrast detection. We recruited a sample of University students ( $n=93$, after exclusions) that were predominantly 18-20 years of age (81/93; see Table S1 for 

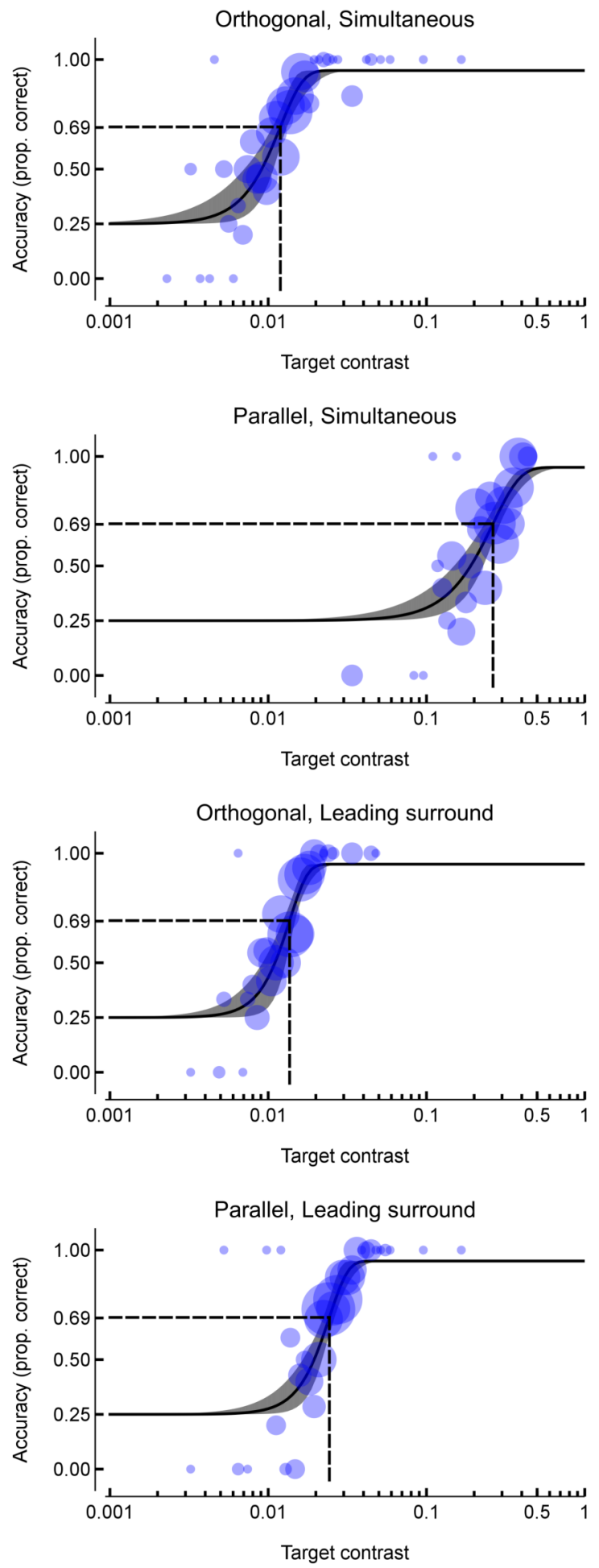

Figure 2. Data and psychometric functions for a representative participant. Circles represent the proportion of correct responses within a given target contrast bin, with an area that is proportional to the number of trials at that contrast. Solid black lines depict the psychometric function, with the grey surrounding region capturing the $95 \%$ CI. The dashed lines indicate the contrast detection threshold (the target contrast corresponding to $69.25 \%$ accuracy). Vertical axes are accuracy (proportion correct) and the horizontal axes are the target contrast (logarithmic spacing). 

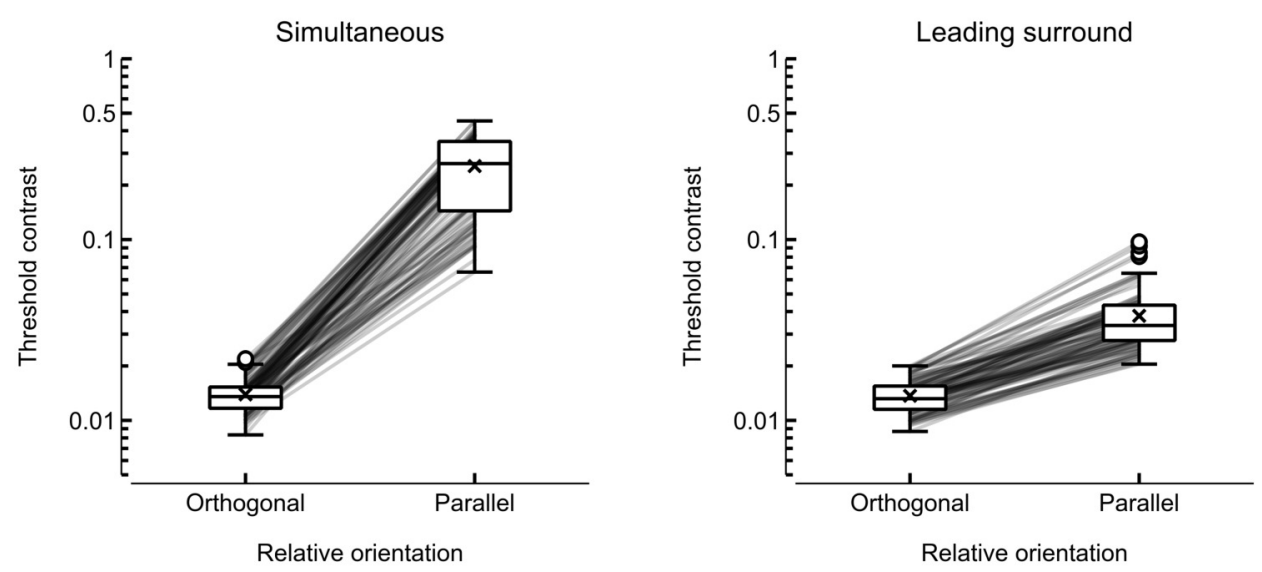

Figure 3. Contrast detection thresholds across the four conditions. The left and right panels show the conditions in which the context was presented with the same (simultaneous) or different (leading surround) temporal schedule as the target. The top and bottom of a given box are the 75th and 25th percentile scores, respectively, with the dividing horizontal line at the median (50th percentile). The whiskers extend as far as the score that is within 1.5 of the inter-quartile range, and any scores outside the whiskers are marked with a circle. The crosses indicate the means of the distributions. Each line connects the thresholds for orthogonal and parallel context for a given participant.

the complete age distribution), female (63/93), and right-handed (91/93). The distribution of O-LIFE subscale scores are qualitatively similar to the norms reported by Mason and Claridge (2006), with the exception of the "unusual experiences" subscale which is lower in the current sample (see Figure S2). The pairwise correlations between the O-LIFE subscales are shown in Figure S3.

\section{Detection thresholds}

The experimental paradigm was motivated by previous reports of an orientation-dependent contextual effect on contrast detection thresholds (Petrov et al., 2005, for example). First, we evaluated whether this effect was evident in the current data. As seen in Figure 3, a simultaneously-presented parallel context resulted in a large increase in the contrast detection threshold relative to a simultaneously-presented orthogonal context; from a mean of $1.39 \%(95 \% \mathrm{CI}[1.33,1.45])$ with orthogonal context to a mean of $25.49 \%(95 \%$ CI $[23.23,27.71])$ with parallel context. Presenting the surrounding context slightly earlier in time than the target led to a much more moderate increase; from a mean of $1.37 \%$ (95\% CI $[1.31,1.43])$ with orthogonal context to a mean of $3.79 \%(95 \%$ CI $[3.49,4.11])$ with parallel context. Hence, the current experimental paradigm was able to invoke orientation-dependent contextual modulation of contrast detection thresholds and to moderate the effect by changing the temporal schedule of the stimulation. We now consider the aims of the current study - to examine if the variabilities in the above effects are related to schizotypy.

\section{Relationship between contextual modulation and schizotypy for simultaneous presenta- tion}

We hypothesised that the influence of a parallel surround on the ability to perceive a target pattern, relative to an orthogonal surround, would reduce with increasing levels of psychometrically-defined schizotypy. To test this hypothesis, we compared the ranked difference in contrast detection thresholds for parallel and orthogonal surrounds with the ranked scores on each of the subscales of the O-LIFE questionnaire (see Figure S4 for a comparison of unranked data). Contrary to our hypothesis, there was no apparent relationship between the relative magnitude of orientation-dependent contextual modulation and relative score on any of the O-LIFE subscales, as shown in Figure 4. Quantifying the monotonicity of this relationship yielded correlation coefficients that were not significantly different from zero for any of the O-LIFE subscales ("unusual experiences": $r=0.02, p=.821,95 \%$ CI $[-0.18,0.23]$; "cognitive disorganisation": $r=-0.04, p=.681,95 \% \mathrm{CI}[-0.25,0.17]$; "introvertive anhedonia": $r=-0.09$, $p=.406,95 \%$ CI $[-0.30,0.13]$; "impulsive nonconformity": $r=0.03, p=.766,95 \%$ CI $[-0.18,0.23]$ ). 


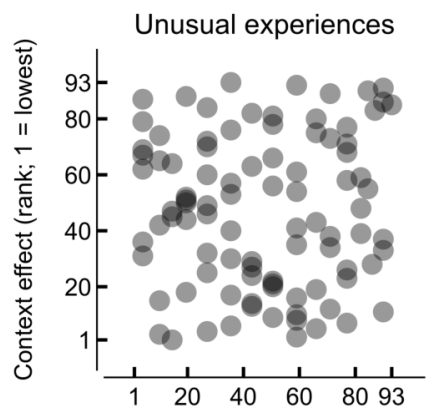

O-LIFE subscale (rank; 1 = lowest)

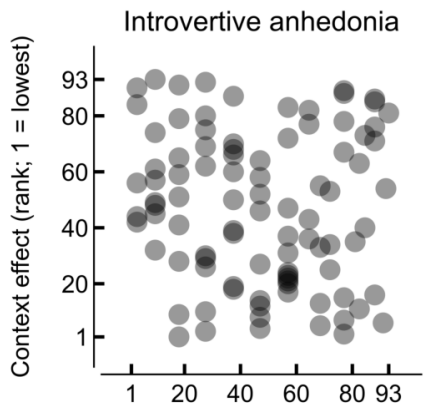

O-LIFE subscale (rank; 1 = lowest)

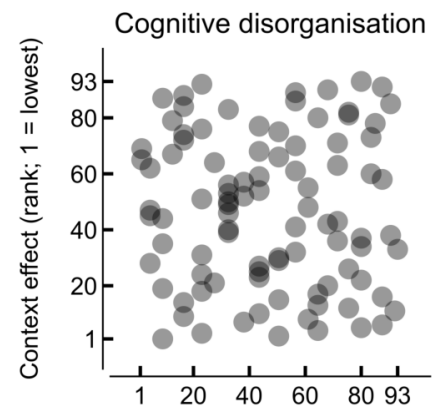

O-LIFE subscale (rank; 1 = lowest)

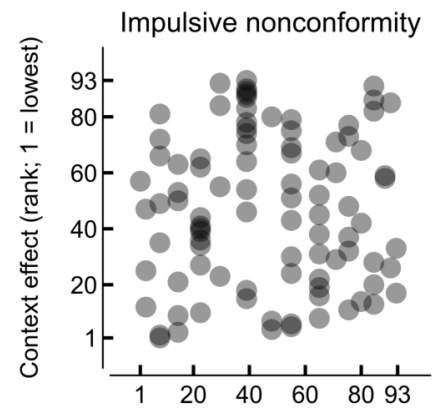

O-LIFE subscale (rank; 1 = lowest)

Figure 4. Comparison of ranked O-LIFE score and the ranked magnitude of the orientation-dependent effect of context during simultaneous presentation. Each point shows a single participant's O-LIFE subscale rank and orientation-dependent context effect (obtained by ranking the difference between the contrast detection threshold with a parallel surround and that with an orthogonal surround, both during simultaneous presentation). 


\section{Relationship between schizotypy and detection thresholds for orthogonal simultaneous context}

Our next hypothesis was that the predicted reduction in orientation-dependent contextual modulation of contrast detection thresholds in those that scored highly on psychometrically-defined schizotypy would be due to elevated contrast detection thresholds with an orthogonal context. This would be expressed as a positive relationship between the ranked scores on psychometrically-defined schizotypy and the ranked contrast detection thresholds in the orthogonal context condition with simultaneous presentation. However, we found little evidence for a monotonic relationship between these measures on any of the O-LIFE subscales ("unusual experiences": $r=0.07, p=.521,95 \%$ CI $[-0.15,0.27]$; "cognitive disorganisation": $r=-0.05, p=.646,95 \% \mathrm{CI}[-0.26,0.16]$; "introvertive anhedonia": $r=-0.11$, $p=.314,95 \% \mathrm{CI}[-0.30,0.10]$; "impulsive nonconformity": $r=0.04, p=.724,95 \% \mathrm{CI}[-0.18,0.24])$. These relationships are shown in Figure S5.

\section{Relationship between schizotypy and temporal influences on contextual modulation}

An additional aim of this experiment was to investigate the temporal characteristics of orientationdependent contextual modulation and its relationship with psychometrically-defined schizotypy. In particular, we hypothesised that presenting the context slightly earlier in time than the target would yield an increased influence of a parallel context, relative to an orthogonal context, for those that scored highly on psychometrically-defined schizotypy. Accordingly, we examined the relationship between the ranked level of psychometrically-defined schizotypy and the relative magnitude of the difference between contrast detection thresholds with parallel and orthogonal context for the 'leading surround' condition. However, there was no discernible relationship between these measures on any of the O-LIFE subscales ("unusual experiences": $r=0.14, p=.177,95 \% \mathrm{CI}[-0.08,0.36]$; "cognitive disorganisation": $r=-0.01, p=.933,95 \%$ CI $[-0.22,0.21]$; "introvertive anhedonia": $r=-0.14, p=.196,95 \% \mathrm{CI}$ $[-0.33,0.07]$; "impulsive nonconformity": $r=0.14, p=.179,95 \%$ CI $[-0.07,0.34])$, as shown in Figure S6.

\section{Bayesian analyses}

The above analyses show that there is a high probability of observing the obtained correlation coefficients if the null hypothesis of no monotonic relationship is true. However, this failure to reject the null hypothesis does not allow for strong conclusions concerning the viability of the null hypothesis itself. To evaluate the strength of the evidence in favour of the null hypothesis, we used the approach of Wetzels and Wagenmakers (2012) to compute Bayes factors for each of the obtained correlations given our sample size $(n=93)$. As shown in Figure 5, the obtained correlation coefficients have Bayes factors that are consistent with the null hypothesis (no monotonic relationship between the given variables); the $\operatorname{logarithm}$ of all of the Bayes factors was $<0$ (highest log Bayes factor $=-1.61$ ). Each of these Bayes factors satisfies the criteria for "substantial" or "strong" support for the null hypothesis, according to the definitions of Wetzels and Wagenmakers (2012).

\section{DISCUSSION}

The primary aim of this study was to determine whether psychometrically-defined schizotypy relates to the extent to which the ability to detect a visual pattern depends on the relative orientation of the target and its surrounding context. Based on previous reports that a diagnosis of schizophrenia is associated with a reduction of the impact of parallel context, relative to orthogonal context (Serrano-Pedraza et al., 2014; Yoon et al., 2009), we hypothesised that individuals scoring highly on psychometrically-defined schizotypy dimensions would be less affected in their ability to detect a vertical pattern with a vertical surround relative to a horizontal surround. However, contrary to our hypothesis, we found no evidence of such a relationship - instead, we found evidence for the absence of such a relationship.

We also investigated whether the threshold for detecting a visual pattern with an orthogonal surround was related to the relative score on psychometrically-defined schizotypy. Previous reports have suggested that those with schizophrenia have elevated thresholds under such presentation conditions (SerranoPedraza et al., 2014; Yoon et al., 2009). However, we again find no evidence of such a relationshipfurthermore, we again found evidence for the absence of such a relationship.

Our secondary aim in conducting this study was to determine whether the effect of the temporal presentation schedule of the target and surround affected visual contrast detection thresholds differently 


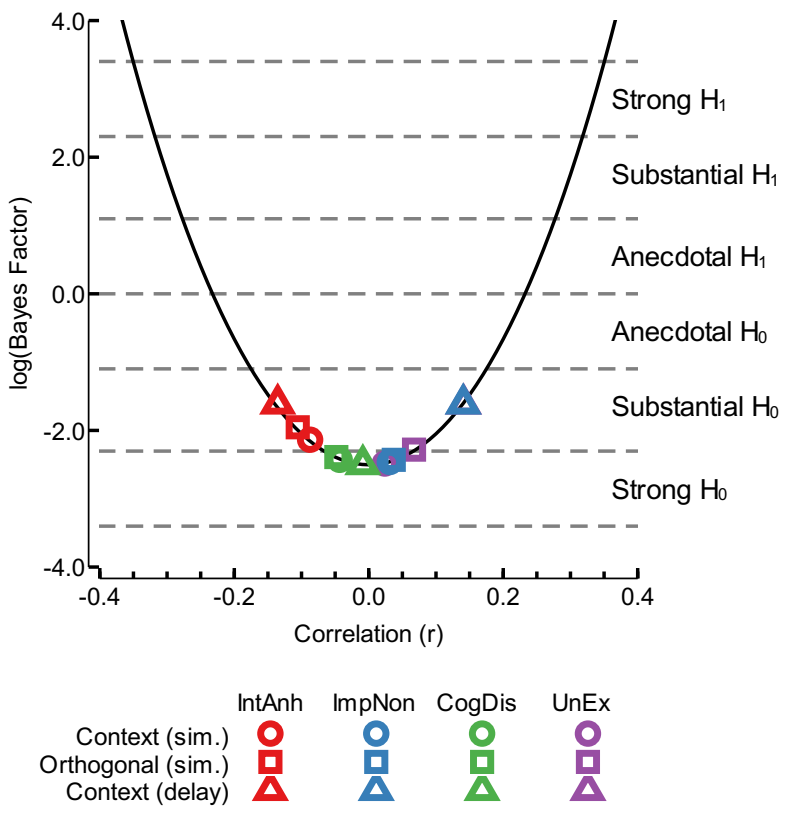

Figure 5. Bayesian analysis of correlation coefficients. We used the approach of Wetzels and Wagenmakers (2012) to determine the relationship between a given correlation coefficient and its Bayes factor, with a fixed sample size of 93 , as depicted by the solid black line. The points show the positioning on this curve of the analyses conducted in this study; different colours depict the dimensions of the schizotypy scale (red: introvertive anhedonia [IntAnh]; blue: impulsive nonconformity [ImpNon]; green: cognitive disorganisation [CogDis]; purple: unusual experiences [UnEx]) and different shapes depict the class of analysis (circles: context effect with simultaneous presentation; squares: orthogonal threshold with simultaneous presentation; triangles: context effect with delayed target presentation). Dashed grey lines denote the Bayes factor interpretation categories, following Wetzels and Wagenmakers (2012). 
depending on the relative degree of psychometrically-defined schizotypy. Specifically, we predicted that those ranked highly on psychometrically-defined schizotypy would be more affected by the slightlyearlier presentation of the surround relative to the target. However, contrary to this hypothesis, we find no evidence of a relationship between psychometrically-defined schizotypy and orientation-dependent contextual modulation when the surround led the presentation of the target-we instead found evidence for the absence of such a relationship.

\section{Implications of the current findings}

The results of the current study are consistent with psychometrically-defined schizotypy being unrelated to the orientation-dependent modulation of visual contrast detection. Given that previous studies report that the extent of such orientation-dependent contextual modulation is related to a diagnosis of schizophrenia (Serrano-Pedraza et al., 2014; Yoon et al., 2009), this suggests that orientation-dependent modulation of visual contrast detection may be a situation in which schizophrenia and psychometrically-defined schizotypy are dissociated. As stated by Ettinger et al. (2015), such situations can be indicative of "protective or compensatory mechanisms" (p. S418) and are hence of considerable interest in understanding the transition to psychosis. Orientation-dependent contextual modulation may be a particularly useful paradigm for revealing such mechanisms due to its well-studied behavioural and neural foundations (Seymour et al., 2013; Yoon et al., 2013) and established theories regarding its circuitry in schizophrenia (Yoon et al., 2010). Should the results and conclusions presented here prove to be robust, future studies using schizotypy may usefully correspond and interact with investigations of schizophrenia to aid in clarifying the mechanisms that selectively relate to the transition to disorder.

\section{Potential limitations of the current study}

However, we must also consider whether there are factors relating to the current study that hamper our ability to draw strong conclusions. As we require the presence of the orientation-dependent contextual modulation of visual contrast detection in order to probe its potential relationship with psychometricallydefined schizotypy, it is important to consider whether this requirement was satisfied in the current experiment. As shown in Figure 3, the presence of parallel context led to a large increase in contrast detection thresholds compared to an orthogonal context - the magnitude of this increase is comparable to that reported by Serrano-Pedraza et al. (2014) using a similar paradigm.

A further important prerequisite is that the sample contained sufficient variation in levels of psychometricallydefined schizotypy. The sampled O-LIFE scores were qualitatively similar to the general-population norms reported by Mason and Claridge (2006), with the exception of the "unusual experiences" subscale in which the current sample was lower than the norms. It is possible that the current sample was insufficiently variable along this dimension to capture a true relationship with orientation-dependent contextual modulation. Furthermore, it is also possible that the current sample contained insufficient participants with extreme scores. Future studies that use a prescreening recruitment strategy may be useful in clarifying the relationship between schizotypy and orientation-dependent contextual modulation.

A desirable component of an investigation of schizotypy, absent in the current study, is the inclusion of a positive control condition - that is, a condition in which there is a strong expectation, based on an accumulation of previous findings, that performance on that condition will be related to psychometricallydefined schizotypy. Recovering such a result in the observed data would provide increased confidence in the validity of the analyses and on the sufficiency of the sample characteristics. However, we suggest that such an approach is, unfortunately, premature for vision and schizotypy - as reviewed in the Introduction, there are currently few (if any) established paradigms that are known to reliably associate visual performance with psychometrically-defined schizotypy. The identification and development of such paradigms is an important area of future research.

Finally, an additional potential limitation of this study (and of related studies) concerns the parameterisation of the surrounding stimulus contrast. Here, the contrast of the surround was fixed (at 25\%) for all participants-similar to the usage of fixed surround contrasts of $25 \%$ and $100 \%$ in Serrano-Pedraza et al. (2014) and Yoon et al. (2009), respectively. When comparing groups with differing contrast sensitivity, such as is evident in those with and without schizophrenia (Serrano-Pedraza et al., 2014), the fixed nature of the surround contrast becomes potentially challenging. This challenge arises because the surround pattern will have different effective contrasts for the groups-for those that are highly sensitive to visual contrast, a surround of a particular stimulus contrast will have a greater effective contrast than it would for those that have reduced contrast sensitivity. Particularly given that the relationship between surround 
contrast and its effect on the perception of the central target is nonlinear (Petrov et al., 2005), it is difficult to identify whether it is changes to the nature of the centre-surround relationship that explain the differing visual performance between groups or whether it instead reflects that the groups are being evaluated at differing locations on a similar centre-surround relationship. These candidate explanations have differing implications for the underlying mechanisms, and we suggest that future studies be devoted to evaluating and resolving this uncertainty - as has been investigated for contextual modulation changes with aging by Karas and McKendrick (2011).

\section{Evaluating the evidence for the effect in schizophrenia}

It is also important to assess the strength of the previously-reported evidence for the differential orientationspecific contextual modulation in schizophrenia before we can confidently assert the distinction between schizophrenia and schizotypy that is suggested by our results. The hypothesis that we entertained in the current study was motivated by previous reports of a different profile of orientation-dependent contextual modulation of contrast perception in schizophrenia - in particular, the studies by Yoon et al. (2009) and Serrano-Pedraza et al. (2014).

The fundamental prediction of the proposed alteration of orientation-dependent contextual modulation of contrast perception in schizophrenia is that there will be an interaction between group (those with and without a diagnosis of schizophrenia) and stimulus condition (parallel and orthogonal context orientation) for the relevant dependent variable. In Yoon et al. (2009), the primary dependent variable was the contrast by which a section of a target annulus had to be reduced for participants to perform with $79 \%$ accuracy (percent correct) on a yes/no task in which they judged whether there was a section of reduced contrast in the target. While the relevant statistical test for the interaction between group and stimulus condition (parallel and orthogonal surround) was not reported in Yoon et al. (2009), a re-analysis of the data indicated that this interaction is statistically significant $\left(F_{1,35}=5.85, p=0.021\right)$. In Serrano-Pedraza et al. (2014), the primary dependent variable was the contrast at which the spatial location of a target could be identified from four alternatives at $62 \%$ accuracy (percent correct). The interaction between group and stimulus condition was not statistically significant, providing no evidence for altered orientation-dependent contextual modulation of contrast perception in schizophrenia. Finally, there is also a relevant study by Schallmo et al. (2015) in which the primary dependent variable was the contrast by which an isolated target needed to be adjusted in order to perceptually match the contrast of an adjacent target that was embedded in an articulated spatial context. The interaction between group (those with and without a diagnosis of schizophrenia) and stimulus condition (parallel and orthogonal surround) was not statistically significant.

The evidence considered thus far for a difference in orientation-dependent contextual modulation of target perception in those with and without a diagnosis of schizophrenia is equivocal - while the data reported in Yoon et al. (2009) show a significant interaction between group (those with and without a diagnosis of schizophrenia) and surround orientation (parallel and orthogonal), the data reported in Serrano-Pedraza et al. (2014) and Schallmo et al. (2015) do not. However, Yoon et al. (2009) and SerranoPedraza et al. (2014) also consider a transformed dependent variable in which the performance with parallel and orthogonal context is expressed relative to performance where the target has no articulated surrounding context. In both cases, statistical analysis on such ratios (or log ratios, in Serrano-Pedraza et al. (2014)) demonstrated a significant interaction between group and stimulus condition-with the parallel ratio significantly larger in those without a diagnosis of schizophrenia and the orthogonal ratio not significantly different in those with and without schizophrenia.

The nature of the dependent variable thus appears to be relevant to the conclusions drawn about the presence of altered orientation-dependent contextual modulation of contrast perception in schizophrenia. The rationale behind the ratio transformation appears to be based on a desire to adjust for differences in performance in the absence of any articulated context. However, interpreting ratios can be challenging due to a critical requirement to be satisfied in order for the transformation to appropriately achieve the desired control without distortion - specifically, the relationship between the numerator and the denominator must be linear and must pass through the origin (Allison et al., 1995; Curran-Everett, 2013). We re-analysed the data of Yoon et al. (2009) and Serrano-Pedraza et al. (2014) to assess these requirements (see Article S1). We find that the requirements were not always satisfied, and that such violations had demonstrable consequences in the Serrano-Pedraza et al. (2014) data while the Yoon et al. (2009) data appeared to be less affected. Hence, we suggest that the comparison between those with and without schizophrenia 
using such transformed data needs to be interpreted with caution (see Curran-Everett, 2013, for further discussion of such issues).

\section{CONCLUSION}

Psychometrically-defined schizotypy appears to be unrelated to the degree to which visual contrast detection thresholds are affected by the relative orientation between the target and its surround. This could identify orientation-specific contextual modulation of visual contrast detection as a paradigm in which schizophrenia is dissociated from psychometrically-defined schizotypy, and hence important in identifying protective or compensatory mechanisms. However, additional research is required to affirm the reported relationship between orientation-dependent contextual modulation and schizophrenia.

\section{ACKNOWLEDGMENTS}

Thanks to Norah Grewal for data collection. We also thank the authors of Yoon et al. (2009), SerranoPedraza et al. (2014), and Schallmo et al. (2015) for sharing the data from their studies.

\section{REFERENCES}

Allison, D. B., Paultre, F., Goran, M. I., Poehlman, E. T., and Heymsfield, S. B. (1995). Statistical considerations regarding the use of ratios to adjust data. International Journal of Obesity and Related Metabolic Disorders, 19(9):644-652.

Asai, T., Sugimori, E., and Tanno, Y. (2008). Schizotypal personality traits and prediction of one's own movements in motor control: what causes an abnormal sense of agency? Consciousness and Cognition, 17(4):1131-1142.

Barbato, M., Collinson, S. L., and Casagrande, M. (2012). Altered depth perception is associated with presence of schizotypal personality traits. Cognitive Neuropsychiatry, 17(2):115-132.

Barch, D. M., Carter, C. S., Dakin, S. C., Gold, J., Luck, S. J., Macdonald, 3rd, A., Ragland, J. D., Silverstein, S., and Strauss, M. E. (2012). The clinical translation of a measure of gain control: the contrast-contrast effect task. Schizophrenia Bulletin, 38(1):135-143.

Burch, G. S., Steel, C., and Hemsley, D. R. (1998). Oxford-Liverpool Inventory of Feelings and Experiences: reliability in an experimental population. British Journal of Clinical Psychology, 37 (Pt 1):107-108.

Butler, P. D., Silverstein, S. M., and Dakin, S. C. (2008). Visual perception and its impairment in schizophrenia. Biological Psychiatry, 64(1):40-47.

Cannon, M. W. and Fullenkamp, S. C. (1991). Spatial interactions in apparent contrast: inhibitory effects among grating patterns of different spatial frequencies, spatial positions and orientations. Vision Research, 31(11):1985-1998.

Cappe, C., Herzog, M. H., Herzig, D. A., Brand, A., and Mohr, C. (2012). Cognitive disorganisation in schizotypy is associated with deterioration in visual backward masking. Psychiatry Research, 200(2-3):652-659.

Curran-Everett, D. (2013). Explorations in statistics: the analysis of ratios and normalized data. Advances in Physiology Education, 37(3):213-219.

Dakin, S., Carlin, P., and Hemsley, D. (2005). Weak suppression of visual context in chronic schizophrenia. Current Biology, 15(20):R822-R824.

Ettinger, U., Mohr, C., Gooding, D. C., Cohen, A. S., Rapp, A., Haenschel, C., and Park, S. (2015). Cognition and brain function in schizotypy: a selective review. Schizophrenia Bulletin, 41 Suppl 2:S417-S426.

Ford, J. M., Mathalon, D. H., Heinks, T., Kalba, S., Faustman, W. O., and Roth, W. T. (2001). Neurophysiological evidence of corollary discharge dysfunction in schizophrenia. American Journal of Psychiatry, 158(12):2069-2071.

Karas, R. and McKendrick, A. M. (2011). Increased surround modulation of perceived contrast in the elderly. Optometry and Vision Science, 88(11):1298-1308.

Kilpeläinen, M., Donner, K., and Laurinen, P. (2007). Time course of suppression by surround gratings: highly contrast-dependent, but consistently fast. Vision Research, 47(26):3298-3306.

Kontsevich, L. L. and Tyler, C. W. (1999). Bayesian adaptive estimation of psychometric slope and threshold. Vision Research, 39(16):2729-2737. 
Koychev, I., El-Deredy, W., Haenschel, C., and Deakin, J. F. W. (2010). Visual information processing deficits as biomarkers of vulnerability to schizophrenia: an event-related potential study in schizotypy. Neuropsychologia, 48(7):2205-2214.

Lenzenweger, M. F. (2010). Schizotypy and schizophrenia: the view from experimental psychopathology. Guilford Press, New York, NY.

Lenzenweger, M. F. and O'Driscoll, G. A. (2006). Smooth pursuit eye movement and schizotypy in the community. Journal of Abnormal Psychology, 115(4):779-786.

Lu, Z.-L. and Dosher, B. (2014). Visual psychophysics. From laboratory to theory. MIT Press, Cambridge, MA.

Mason, O. and Claridge, G. (2006). The Oxford-Liverpool Inventory of Feelings and Experiences (O-LIFE): further description and extended norms. Schizophrenia Research, 82(2-3):203-211.

Mason, O. and Claridge, G. (2015). Schizotypy: New dimensions. Routledge, New York, NY.

Mason, O., Claridge, G., and Jackson, M. (1995). New scales for the assessment of schizotypy. Personality and Individual Differences, 18(1):7-13.

Modinos, G., Mechelli, A., Ormel, J., Groenewold, N. A., Aleman, A., and McGuire, P. K. (2010). Schizotypy and brain structure: a voxel-based morphometry study. Psychol Med, 40(9):1423-1431.

Oestreich, L. K. L., Mifsud, N. G., Ford, J. M., Roach, B. J., Mathalon, D. H., and Whitford, T. J. (2015). Subnormal sensory attenuation to self-generated speech in schizotypy: Electrophysiological evidence for a 'continuum of psychosis'. International Journal of Psychophysiology, 97(2):131-138.

Oestreich, L. K. L., Mifsud, N. G., Ford, J. M., Roach, B. J., Mathalon, D. H., and Whitford, T. J. (2016) Cortical suppression to delayed self-initiated auditory stimuli in schizotypy: Neurophysiological evidence for a continuum of psychosis. Clinical EEG and Neuroscience, 47(1):3-10.

Peirce, J. W. (2007). Psychopy—psychophysics software in Python. Journal of Neuroscience Methods, 162:8-13.

Petrov, Y., Carandini, M., and McKee, S. (2005). Two distinct mechanisms of suppression in human vision. Journal of Neuroscience, 25(38):8704-8707.

Petrov, Y. and McKee, S. P. (2009). The time course of contrast masking reveals two distinct mechanisms of human surround suppression. Journal of Vision, 9(1):21.1-2111.

Rawlings, D. and Claridge, G. (1984). Schizotypy and hemisphere function-III. Personality and Individual Differences, 5(6):657-663.

Schafer, E. W. and Marcus, M. M. (1973). Self-stimulation alters human sensory brain responses. Science, 181(4095):175-177.

Schallmo, M.-P., Sponheim, S. R., and Olman, C. A. (2015). Reduced contextual effects on visual contrast perception in schizophrenia and bipolar affective disorder. Psychological Medicine, 45:3527-3537.

Serrano-Pedraza, I., Romero-Ferreiro, V., Read, J. C. A., Diéguez-Risco, T., Bagney, A., CaballeroGonzález, M., Rodríguez-Torresano, J., and Rodriguez-Jimenez, R. (2014). Reduced visual surround suppression in schizophrenia shown by measuring contrast detection thresholds. Frontiers in Psychology, $5: 1431$

Seymour, K., Stein, T., Sanders, L. L. O., Guggenmos, M., Theophil, I., and Sterzer, P. (2013). Altered contextual modulation of primary visual cortex responses in schizophrenia. Neuropsychopharmacology, 38(13):2607-2612.

Silverstein, S. M., Raulin, M. L., Pristach, E. A., and Pomerantz, J. R. (1992). Perceptual organization and schizotypy. Journal of Abnormal Psychology, 101(2):265-270.

Tibber, M. S., Anderson, E. J., Bobin, T., Antonova, E., Seabright, A., Wright, B., Carlin, P., Shergill, S. S., and Dakin, S. C. (2013). Visual surround suppression in schizophrenia. Frontiers in Psychology, 4:88.

Uhlhaas, P. J., Silverstein, S. M., Phillips, W. A., and Lovell, P. G. (2004). Evidence for impaired visual context processing in schizotypy with thought disorder. Schizophrenia Research, 68(2-3):249-260.

Wetzels, R. and Wagenmakers, E.-J. (2012). A default Bayesian hypothesis test for correlations and partial correlations. Psychonomic Bulletin \& Review, 19(6):1057-1064.

Whitford, T. J., Mathalon, D. H., Shenton, M. E., Roach, B. J., Bammer, R., Adcock, R. A., Bouix, S., Kubicki, M., De Siebenthal, J., Rausch, A. C., Schneiderman, J. S., and Ford, J. M. (2011). Electrophysiological and diffusion tensor imaging evidence of delayed corollary discharges in patients with schizophrenia. Psychological Medicine, 41(5):959-969.

Yang, E., Tadin, D., Glasser, D. M., Hong, S. W., Blake, R., and Park, S. (2013). Visual context processing 
474 in schizophrenia. Clinical Psychological Science, 1(1):5-15.

475 Yoon, J. H., Maddock, R. J., Rokem, A., Silver, M. A., Minzenberg, M. J., Ragland, J. D., and Carter, 476 C. S. (2010). GABA concentration is reduced in visual cortex in schizophrenia and correlates with 477 orientation-specific surround suppression. Journal of Neuroscience, 30(10):3777-3781. (2009). Diminished orientation-specific surround suppression of visual processing in schizophrenia.

$480 \quad$ Schizophrenia Bulletin, 35(6):1078-1084.

481 Yoon, J. H., Sheremata, S. L., Rokem, A., and Silver, M. A. (2013). Windows to the soul: vision science as 482 a tool for studying biological mechanisms of information processing deficits in schizophrenia. Frontiers 483 in Psychology, 4:681.

484 Zenger-Landolt, B. and Heeger, D. J. (2003). Response suppression in V1 agrees with psychophysics of surround masking. Journal of Neuroscience, 23(17):6884-6893. 